\title{
EVALUATION OF WOOD SURFACE COATING PERFORMANCE USING WATER BASED, SOLVENT BASED AND POWDER COATING
}

Ender Haziri,^, Kücük Huseyin Koc ${ }^{2}$

\begin{abstract}
The objective of this study was to evaluate the surface coating performance using water base, solvent base and powder coating on medium density fiberboard. Samples were coated using water-base, solvent-base and powder coating. Coating performance was performed by using adhesion strength, surface coating hardness, layer thickness and rapid deformation test. The results were analyzed with two different statistical methods. Adhesion strength, layer thickness and surface hardness were determined by using ANOVA analysis, while results of rapid deformation test were analyzed by using Kruskal-Wallis method. According to these results, coating type was effective factor on the adhesion strength, surface coating hardness, layer thickness and rapid deformation test. Water-based painting application for adhesion strength and impact deformation resistance were higher than solvent-based coating. In the powder coating application, although surface coating hardness was higher than solvent and water base coating application, rapid deformation results were lower than solvent and water base coating applications. Adequacy of models was performed $R$-square $\left(R^{2}\right)$ and Adjusted $R$-square $\left(\right.$ Adj- $R^{2}$ ) values. $R^{2}$ values of adhesion strength, layer hardness and film layer thickness were 93,60\%; 95,33 $\%$ and $73,90 \%$, respectively. Adj- $R^{2}$ values of adhesion strength, layer hardness and film layer thickness were $93,45 \% ; 95,23 \%$ and $73,30 \%$, respectively.
\end{abstract}

Keywords: Adhesion strength, coating hardness, coating thickness, layer hardness, medium-density fiberboard.

\section{INTRODUCTION}

Particleboard, fiberboard and wood based materials are widely used in furniture industry and cabinet production. Surface quality and type of the coating material are two significant factors influencing overall quality of the product (Ahola 1991, De Moura and Hernandez 2006, Ramananantoandro et al. 2018, Salca et al. 2017). It is a well-known fact that coating performance namely adhesion is depended on hygroscopic characteristic of wood composite panels. Therefore, coating type plays an important role on coating performance such as good bonding, coating hardness, abrasion resistance, UV resistance and film thickness (Salca et al. 2016 Sogutlu et al. 2016, Nejad and Cooper 2011, Darmawan et al. 2018, Erdinler et al. 2019). Coating applications of varnishes, stain and painting are the most popular method in order to enhance the stability and appearance of the wood and wood based materials. Surface coatings, as one of the final stages of production in wooden furniture and decoration applications, constitute the majority of production cost. In addition to this, it is effective in the performance of the products and the preferences of the customers. Moreover, these applications extend the service life. A good surface finishing increases the usage properties of the product (Cool and Hernandez 2011,

\footnotetext{
${ }^{1}$ Faculty of Forestry, Department of Forest Industry Engineering, Istanbul University - Cerrahpasa, Istanbul, Turkey. ender.hazir istanbul. edu.tr

${ }^{2}$ Faculty of Forestry, Department of Forest Industry Engineering, Istanbul University-Cerrahpasa, Istanbul, Turkey. hkoc@istanbul.edu.tr "Corresponding author: ender.hazir@istanbul.edu.tr

Received: 26.04.2018 Accepted: 27.05.2019
} 
Landry et al. 2013). Medium density fiberboard and particleboards gradually increases in furniture and decoration applications due to covering surface treatments. Although there are various national and international standards of surface coatings and their characteristics, it's seen that the required importance is not considered in application and the controls remain at subjective evaluation level. Therefore, in the majority of the applications, the surface coatings may cause an increase in the production costs, prevent achieving the desired surface performance values and decreased surface coating performance (Acda et al. 2012, Nejad et al. 2012, Dilik et al. 2015, Gurleyen et al. 2017).

Hernandez and Cool (2008) evaluated three surfacing methods on paper birch wood in relation to water and solvent borne coating performance. Coating performance was determined by using pull-off strength test. The results showed that the water base coating of adhesion strength was higher than solvent borne coating.

Jocham et al. (2012) used pre-heating process in order to improve the powder application during the powder coating process for MDFs. According to the results, board temperature and moisture content were effective parameters to improve the adhesion strength.

Keskin and Tekin (2011) determined the abrasion resistance of cellulosic, synthetic, polyurethane, waterborne and acid hardening varnishes for Scota pine, Oriental beech, European oak, Black poplar, Basswood and Black walnut woods. It was found that varnish type and amount of layer thickness were significant factors for abrasion resistance.

Cakicier et al. (2011) studied the effects of different heat treatment and varnish coating on hardness, scratch resistance and glossiness of wood materials such as limba, iroka, ash and chestnut. In the different heat treatment application, coating hardness and scratch resistance decreased for the all wood species.

Rield et al. (2014) used to determine the effect of atmospheric pressure plasma treatment on wood surface coating performance using contact angle measurement and pull off testing. The results showed that type of plasma gases and treatment time were significant factors on the wettability and adhesion strength for maple and spruce wood samples.

Dilik et al. (2015) evaluated adhesive strength of particleboard and medium density fiberboard (MDF) finished with different types of paints such as cellulosic and polyurethane based paints. The highest adhesion strength value of 3,62 MPa was found for polyurethane coated MDF samples.

\section{MATERIALS AND METHODS}

\section{Experimental procedure}

This experimental procedure involved four steps in order to determine the coating performance. These steps were:

The coating factors were selected as painting type such as water base, solvent base and powder coating. Adhesion strength, surface hardness, layer thickness and rapid-deformation test were used to determine the coating performance.

Grubb's test was used to determine the outlier value. Analysis for normal distribution data were performed by using probability plot with $95 \%$ interval confidence. One-way ANOVA analysis was applied to determine the significant parameters. Tukey test was used to determine the difference between the groups. Rapid deformation test was evaluated with Kruskal-Wallis method.

ANOVA analysis was performed by using R-square $\left(R^{2}\right)$ and adjusted coefficient of ( $R^{2}$-adj) values.

Adequacy of developed model was tested by performing normal probability plot (NPP) and histogram of residuals. 


\section{Preparation of wood-based material}

MDF (medium density fiberboard) was selected as test material in order to determine the coating performance. A total of 90 specimens with dimensions of $100 \mathrm{~mm}$ by $100 \mathrm{~mm}$ by $18 \mathrm{~mm}$ were conditioned in climate at $(20 \pm 2)^{\circ} \mathrm{C}$ and $(65 \pm 5) \%$ relative humidity $(\mathrm{RH})$ until they reach a moisture content of $(10 \pm 1) \%$. Density value of MDF was measured as $0,76 \mathrm{~g} / \mathrm{cm}^{3}$. The experiments were conducted on a sanding process known as belt sanding machine. Open coat aluminum oxide abrasive type was used to prepare the samples. The MDF materials were sanded with feed rate of $5 \mathrm{~m} / \mathrm{min}$, cutting speed of $18 \mathrm{~m} / \mathrm{sec}$ and grit size of 150 as belt tension of $3 \mathrm{~kg} / \mathrm{cm}^{2}$.

\section{Preparation of wood coating surface}

In this study, three different coating materials such as water-based, solvent-based and powder coating applications were applied on the MDF samples. MDF samples were conditioned in a climate room having a temperature of $20^{\circ} \mathrm{C}$ and relative humidity of $65 \%$ until they reach a moisture content of $8-10 \%$ before they were coated with various types of finishes. In the water-base and solvent-based coating application, specimens were coated with a pneumatic-spray gun using a pressure of $0,80 \mathrm{MPa}$. Each of the surface panels with an angle of 90 degrees were applied primer and top coat of the finishes.

In the powder coating application, the MDF surfaces were not subjected to any chemical pretreatments. The application of heat prior to application provides an even earthing of the substrate for deposition of the powder. After the MDF board was pre-conditioned with convection heat 3 mins at $120^{\circ} \mathrm{C}$, the samples were stoved on the air temperature with 10 minutes at $140^{\circ} \mathrm{C}$. In the powder coating system, application of primer coating thickness and top coating thickness was applied 80-120 $\mathrm{m \mu}$ and 70-100 $\mathrm{m} \mu$, respectively.

\section{Evaluation of the coating performance}

\section{Adhesion strength measurement}

Adhesion strength is one of the most significant indicator for determining the coating performance. It is affected the good coating, resistance and durability performance. In this study, pull-off test method was used to determine the adhesive performance. The test method was carried out EN ISO 4624-2016.

\section{Coating hardness measurement}

Coating hardness is a significant quality characteristic for evaluating the behavior of the finished product during its transport and manufacturing process. Hardness effects many various properties such as impact resistance, stretches and abrasion effects for final products. In this study, EN ISO 1522-2016 method was applied to measure the samples. It is a damping method to evaluate the coating hardness equipped with konig pendulum apparatus.

\section{Film thickness measurement}

Film thickness is one of the important index of coating performance and appearance. As wood and wood based material have a complex structure, values of film thickness measurement have a different variance. In this study, the layer thicknesses of the coats were measured with PosiTector. This equipment is a non-destructive method and it measure the layer thickness of between 20 and $300 \mathrm{~m} \mu$.

\section{Rapid-deformation test}

Rapid-deformation tests evaluate the effects of accidental contact damage that may occur during use. They are usually carried out by the direct evaluation of the effect of a falling object, sphere or dart of specified shape and hardness, onto the tested surface form different heights. During impact on coated surfaces, the deformation of the substrate can be relevant, significantly affecting the final results. It is of importance that the coating film is flexible enough to withstand, to a certain extent, the stretching at the fringes of the deformation without any cracking or cleavage. In this study, EN ISO 4211-4-2017 test was used to measure the specimens. 


\section{Statistical procedure}

In this study, in order to determine the quality characteristics of adhesion strength, coating hardness, layer thickness and impact deformation test, (30x3) measurements were taken for each quality characteristic. The Anderson-Darling test is used to test if a sample of data came from a population with a specific distribution. Grubb's method was used to determine the outlier analyzes. This test detects outliers from normal distributions. The test can detect one outlier at a time with different probabilities from a data set with assumed normal distribution. Analysis of variance and Tukey's test were applied to evaluate the effective parameters.

\section{Analysis of variance}

Analysis of variance (ANOVA) based $F$-test was applied to evaluate the significance factor on the coating performance. This analysis investigates the following for each parameter are given in Equation 1 and Equation 2:

$$
\left.\begin{array}{l}
H_{0}: \mu_{1}=\mu_{2}=\ldots=\mu_{\alpha} \\
H_{1}: \mu_{i} \neq \mu_{j} \text { for at least one pair }(i, j)
\end{array}\right\}
$$

The $F$ value is calculated by:

$$
F_{0}=\frac{S S_{A} / \alpha-1}{S S_{E} / N-\alpha}=\frac{M S_{A}}{M S_{E}}
$$

The terms of $(\alpha-1)$ and $(\mathrm{N}-\alpha)$ are the degrees of freedom and the error degrees of freedom for the parameter A, respectively. $M S_{A}$ and $M S_{E}$ are indicated the sum squares of means and errors for the variable A, respectively. The null hypothesis is rejected when the $F_{0}$ is higher than critical value of $F_{\alpha, \alpha-1, N-\alpha,}$, where $\alpha$ is the level of the significance .

\section{Tukey's test}

Tukey's multiple comparison test is one of several tests that can be used to determine which means amongst a set of means differ from the rest. It is to use a one-way analysis of variance (ANOVA) to evaluate whether there is any evidence that the means of the populations differ. Tukey multiple comparison test assumes that the data from the different groups come from populations where the observations have a normal distribution and the standard deviation is the same for each group.

\section{Non-Parametric test}

Kruskal-Wallis test is a nonparametric test, and is used to categorical independent variable for two or more groups. Kruskal-Wallis test can be used for both continuous and ordinal-level dependent variables. The Kruskal-Wallis test statistic for $\mathrm{k}$ samples, each of size $\mathrm{n}_{\mathrm{i}}$ is given in Equation 3 and Equation 4:

$$
\begin{gathered}
T=\frac{1}{s^{2}}\left[\sum_{i=1}^{k} \frac{R_{i}}{n_{i}}-N \frac{(N+1)^{2}}{4}\right] \\
S^{2}=\frac{1}{N-1}\left[\sum_{\text {all }} R_{j}{ }^{2}-N \frac{(N+1)^{2}}{4}\right]
\end{gathered}
$$

Where $\mathrm{N}$ is the total number (all $\mathrm{n}_{\mathrm{i}}$ ) and $\mathrm{R}_{\mathrm{i}}$ is the sum of the ranks (from all samples pooled) for the each sample. 


\section{RESULTS AND DISCUSSION}

The results of adhesion strength, layer thickness and coating hardness for solvent base, water base and powder coating are given in the (Table 1). For each type of coating types, $30 \times 3$ arrays were measured and these results were evaluated with ANOVA analysis. In order to reduce the variation during measurement, the measurements were performed in a random order for determining the coating performance.

Table 1: Coating performance results for water based, solvent based and powder coating.

\begin{tabular}{|c|c|c|c|c|c|c|c|c|}
\hline \multicolumn{2}{|c|}{ Water base } & \multirow[b]{2}{*}{ Thickness } & \multicolumn{2}{|c|}{ Solvent base } & \multirow[b]{2}{*}{ Thickness } & \multicolumn{2}{|c|}{ Powder coating } & \multirow[b]{2}{*}{ Thickness } \\
\hline Adhesion & Hardness & & Adhesion & Hardness & & Adhesion & Hardness & \\
\hline 3,24 & 288 & 162 & 2,11 & 234 & 159 & 2,78 & 322 & 189 \\
\hline 3,16 & 286 & 155 & 2,15 & 241 & 155 & 2,64 & 317 & 193 \\
\hline 3,47 & 291 & 159 & 2,21 & 225 & 163 & 2,59 & 327 & 191 \\
\hline 2,95 & 278 & 169 & 2,18 & 231 & 174 & 2,71 & 328 & 188 \\
\hline 3,14 & 293 & 162 & 2,13 & 246 & 177 & 2,56 & 342 & 193 \\
\hline 3,27 & 290 & 160 & 2,25 & 240 & 164 & 2,73 & 333 & 182 \\
\hline 3,18 & 301 & 173 & 2,18 & 233 & 168 & 2,68 & 327 & 185 \\
\hline 3,01 & 298 & 165 & 2,14 & 214 & 179 & 2,76 & 329 & 190 \\
\hline 3,09 & 278 & 168 & 2,31 & 241 & 175 & 2,54 & 319 & 193 \\
\hline 3,11 & 274 & 174 & 2,28 & 237 & 164 & 2,62 & 327 & 188 \\
\hline 3,23 & 283 & 164 & 2,31 & 231 & 169 & 2,69 & 331 & 186 \\
\hline 3,31 & 284 & 163 & 2,35 & 229 & 184 & 2,72 & 337 & 186 \\
\hline 3,25 & 281 & 171 & 2,31 & 216 & 184 & 2,59 & 338 & 190 \\
\hline 2,98 & 291 & 160 & 2,3 & 213 & 158 & 2,81 & 330 & 188 \\
\hline 3 & 302 & 152 & 2,2 & 220 & 154 & 2,83 & 318 & 193 \\
\hline 3,32 & 299 & 158 & 2,01 & 230 & 158 & 2,79 & 322 & 189 \\
\hline 3,17 & 297 & 160 & 2,06 & 234 & 174 & 2,66 & 325 & 185 \\
\hline 3,29 & 293 & 172 & 2,11 & 226 & 179 & 2,82 & 329 & 188 \\
\hline 3,24 & 288 & 164 & 2,08 & 216 & 153 & 2,59 & 320 & 186 \\
\hline 3,22 & 285 & 165 & 2,15 & 223 & 154 & 2,68 & 319 & 188 \\
\hline 3,16 & 273 & 160 & 2,17 & 221 & 155 & 2,75 & 316 & 191 \\
\hline 3,18 & 284 & 154 & 2,24 & 220 & 160 & 2,79 & 332 & 183 \\
\hline 3,03 & 281 & 164 & 2,18 & 241 & 171 & 2,88 & 337 & 184 \\
\hline 3,12 & 294 & 170 & 2,24 & 252 & 165 & 2,84 & 339 & 182 \\
\hline 3,46 & 303 & 164 & 2,26 & 246 & 169 & 2,78 & 330 & 185 \\
\hline 2,89 & 296 & 157 & 2,21 & 249 & 173 & 2,81 & 329 & 189 \\
\hline 3,1 & 289 & 169 & 2,16 & 236 & 165 & 2,79 & 326 & 188 \\
\hline 3,28 & 279 & 168 & 2,19 & 238 & 166 & 2,67 & 319 & 184 \\
\hline 3,25 & 300 & 164 & 2,2 & 234 & 170 & 2,6 & 323 & 186 \\
\hline 3,17 & 281 & 169 & 2,21 & 225 & 165 & 2,82 & 326 & 188 \\
\hline
\end{tabular}

\section{Analysis of adhesion strength, layer thickness and coating hardness}

Probability plot of coatings adhesion strength values of for solvent base, water base and powder coating is displayed in (Figure 1). This plot was presented with results of mean, standard deviation (StDev), sample size (N), Andersen darling test (AD) and $P$-critical values. According to the results of $95 \%$ confidence level, $P$-values were high than 0,05 showing the model the observations have a normal distribution. Water-based, 
powder coating and solvent-based mean values for adhesion strength were found as 3,176;2,717 and 2,196 $\mathrm{MPa}$ respectively.

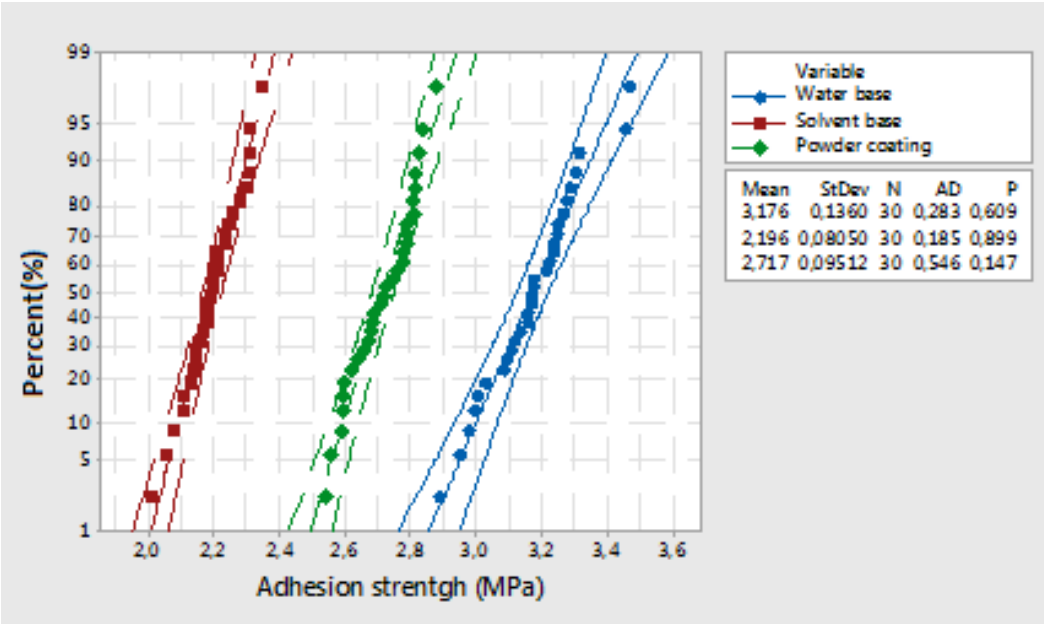

Figure 1: Probability plot of coatings adhesion strength values of for solvent-based, water- based and powder coating.

Probability plot of coatings hardness values of for solvent base, water base and powder coating is displayed in (Figure 2). Powder coating, water-based and solvent-based mean values for coating hardness were found as 327,2; 288,7 and 231,4; respectively. In (Figure 3), powder coating, water-based and solvent-based mean values for coating layer thickness values were found as 187,$7 ; 166,8$ and $163,8 \mathrm{~m} \mu$ respectively.

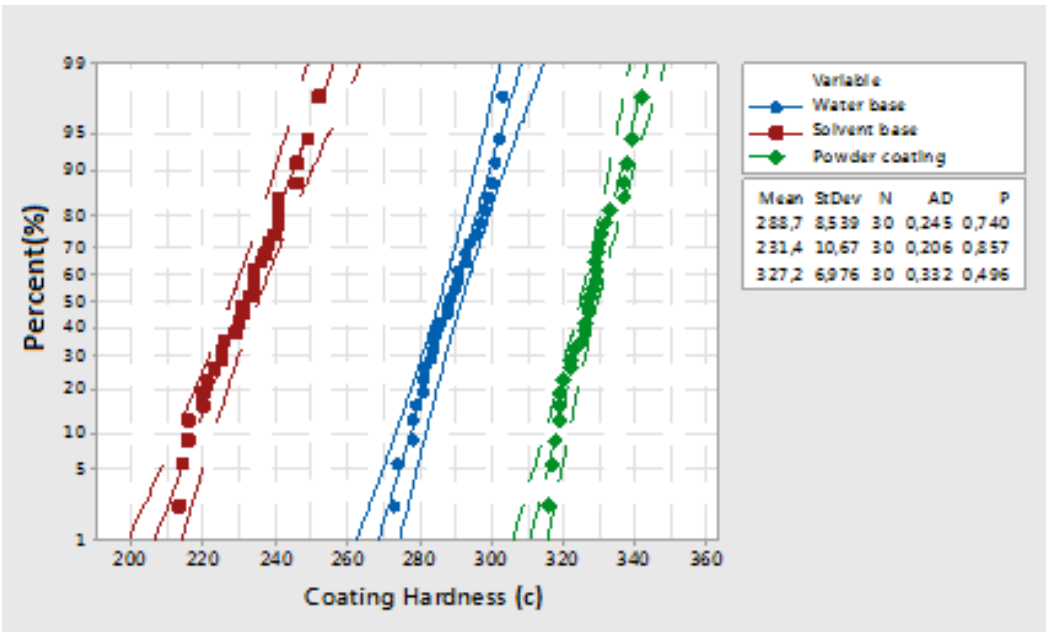

Figure 2: Probability plot of coatings adhesion strength values of for solvent base, water base and powder coating. 


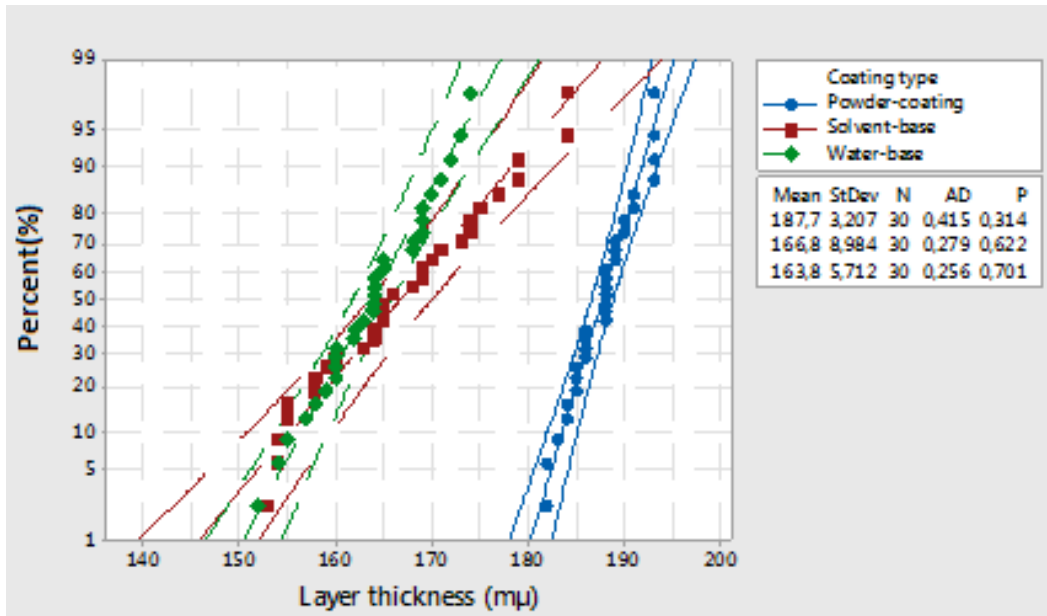

Figure 3: Probability plot of coatings layer thickness values of for solvent base, water base and powder coating.

Outlier test results are given in Table 2, Table 3 and Table 4. These results were presented with results of sample size (N), standard deviation (StDev), minimum value (Min), maximum value (Max), Grubb's test (G) and $P$-critical values. According to the results, at the 5\% level of significance was not outlier values for adhesion strength, layer thickness and coating hardness.

Table 2: Result of outlier test for adhesion strength.

\begin{tabular}{|c|c|c|c|c|c|c|c|}
\hline Variable & N & Mean & StDev & Min & Max & G & P \\
\hline Water base & 30 & 3,1757 & 0,1360 & 2,8900 & 3,4700 & 2,16 & 0,746 \\
\hline Solvent base & 30 & 2,1960 & 0,0805 & 2,0100 & 2,3500 & 2,31 & 0,477 \\
\hline Powder coating & 30 & 2,7173 & 0,0951 & 2,54 & 2,88 & 1,86 & 1,000 \\
\hline
\end{tabular}

Table 3: Result of outlier test for coating hardness.

\begin{tabular}{|c|c|c|c|c|c|c|c|}
\hline Variable & N & Mean & StDev & Min & Max & G & P \\
\hline Water base & 30 & 288,67 & 8,54 & 273 & 303 & 1,83 & 1,000 \\
\hline Solvent base & 30 & 231,40 & 10,67 & 213 & 252 & 1,93 & 1,000 \\
\hline Powder coating & 30 & 327,23 & 6,98 & 316 & 342 & 2,12 & 0,858 \\
\hline
\end{tabular}

Table 4: Result of outlier test for layer thickness.

\begin{tabular}{|c|c|c|c|c|c|c|c|}
\hline Variable & N & Mean & StDev & Min & Max & G & P \\
\hline Water base & 30 & 163,83 & 5,71 & 152 & 174 & 2,07 & 0,975 \\
\hline Solvent base & 30 & 166,80 & 8,98 & 153 & 184 & 1,91 & 1,000 \\
\hline Powder coating & 30 & 187,70 & 3,21 & 182 & 193 & 1,78 & 1,000 \\
\hline
\end{tabular}


Table 5, Table 6 and Table 7 displayed the $P$-value is less than 0,05 showing the model is significant at $95 \%$ confidence level. All the terms of model were tested and verified statistically by $F$-test at probability levels $(p<0,05)$. Adequacy of model for adhesion strength was tested by performing $R^{2}$ and Adj- $R^{2}$ values were $93,60 \%$ and $93,45 \%$, respectively. Adequacy of model for coating hardness was tested by performing with $R^{2}$ and Adj- $R^{2}$ values were 95,33\% and 95,23\%, respectively. Adequacy of model for layer thickness was tested by performing with $R^{2}$ and Adj- $R^{2}$ values were $73,90 \%$ and $73,30 \%$, respectively.

Table 5: ANOVA analysis for adhesion strength.

\begin{tabular}{|c|c|c|c|c|c|}
\hline Source & DF & Adj SS & Adj MS & F-Value & P-Value \\
\hline Paint type & 2 & 14,4160 & 7,20802 & 635,72 & 0,000 \\
\hline Error & 87 & 0,9864 & 0,01134 & & \\
\hline Total & 89 & 15,4025 & & & \\
\hline
\end{tabular}

Table 6: ANOVA analysis for surface coating hardness.

\begin{tabular}{|c|c|c|c|c|c|}
\hline Source & DF & Adj SS & Adj MS & F-Value & P-Value \\
\hline Paint type & 2 & 139509 & 69754,4 & 888,63 & 0,000 \\
\hline Error & 87 & 6829 & 78,5 & & \\
\hline Total & 89 & 146338 & & & \\
\hline
\end{tabular}

Table 7: ANOVA analysis for layer thickness.

\begin{tabular}{|c|c|c|c|c|c|}
\hline Source & DF & Adj SS & Adj MS & F-Value & P-Value \\
\hline Paint type & 2 & 10152 & 5076,14 & 123,18 & 0,000 \\
\hline Error & 87 & 3585 & 41,21 & & \\
\hline Total & 89 & 13748 & & & \\
\hline
\end{tabular}

In Table 8, Table 9 and Table 10, dual comparisons were performed employing Tukey's test. These results show that two pairs of each group were compared and the mean difference between the groups was given numerically. These numerical values indicated the significant difference between the averages of these factors for adhesion strength, layer thickness and coating hardness.

Table 8: Tukey's test results for adhesion strength.

\begin{tabular}{|c|c|c|c|}
\hline Factor & N & Mean & Grouping \\
\hline Water base & 30 & 3,1757 & A \\
\hline Powder coating & 30 & 2,7173 & B \\
\hline Solvent base & 30 & 2,1960 & C \\
\hline
\end{tabular}

Table 9: Tukey's test results for surface coating hardness.

\begin{tabular}{|c|c|c|c|}
\hline Factor & N & Mean & Grouping \\
\hline Powder coating & 30 & 327,23 & A \\
\hline Water base & 30 & 288,67 & B \\
\hline Solvent base & 30 & 231,40 & C \\
\hline
\end{tabular}


Table 10: Tukey's test results for layer thickness.

\begin{tabular}{|c|c|c|c|}
\hline Factor & N & Mean & Grouping \\
\hline Powder coating & 30 & 187,7 & A \\
\hline Solvent base & 30 & 166,8 & B \\
\hline Water base & 30 & 163,8 & B \\
\hline
\end{tabular}

\section{Boxplot and Interval plot}

The box plot is a standardized way of displaying the distribution of data based on the five number summary: minimum, first quartile, median, third quartile, and maximum. The interval plot shows a $95 \%$ confidence interval for the mean of each group. From the (Figure $4 \mathrm{a}$ and Figure $4 \mathrm{~b}$ ), water-based adhesion strength value was higher than solvent-based and powder coating values. In the (Figure 4c and Figure 4d), powder coating surface hardness value was higher than solvent-based and water-based coatings. In the (Figure $4 \mathrm{e}$ and Figure 4f), powder coating layer thickness value was higher than solvent-based and water-based coatings.
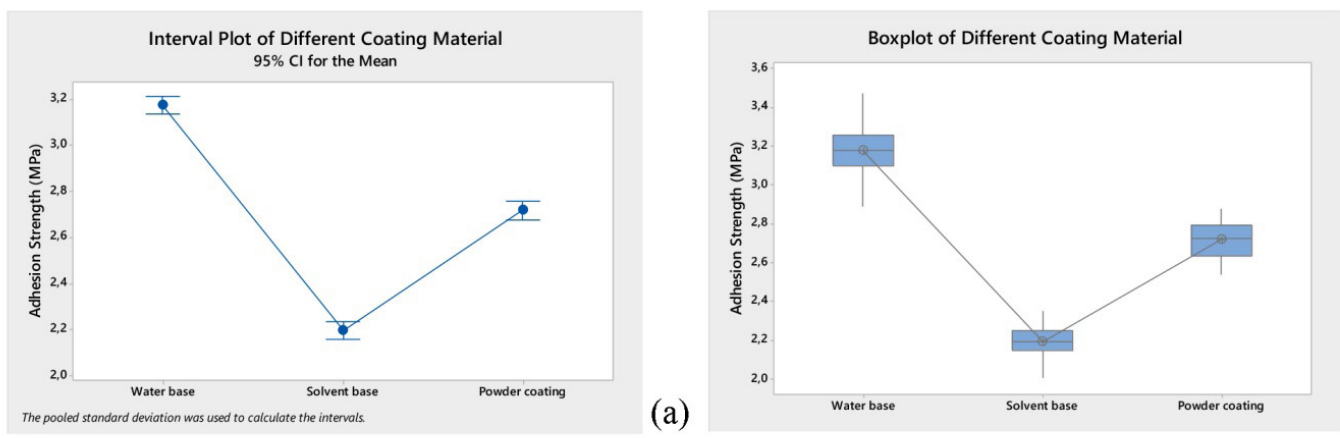

(b)
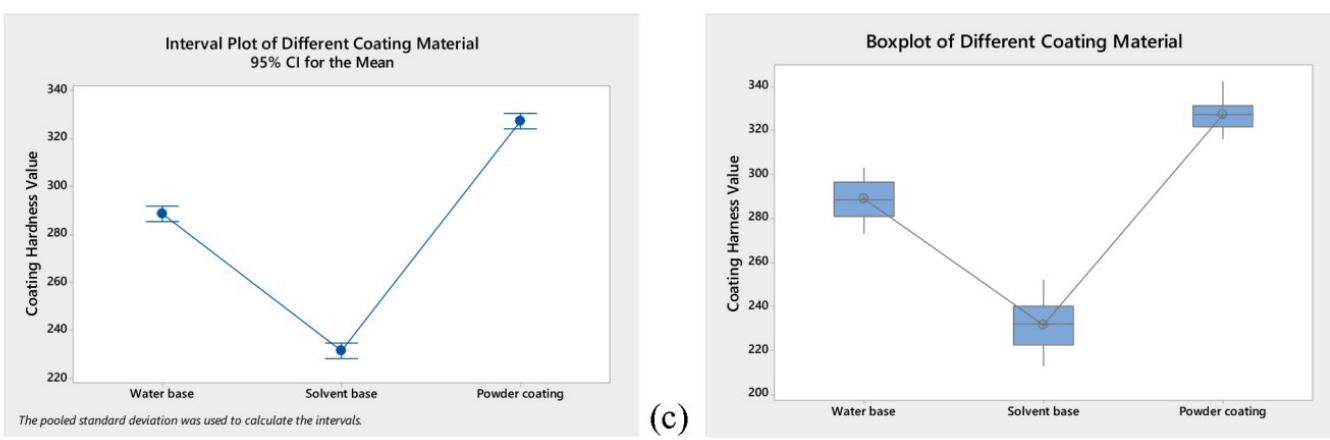

(d)
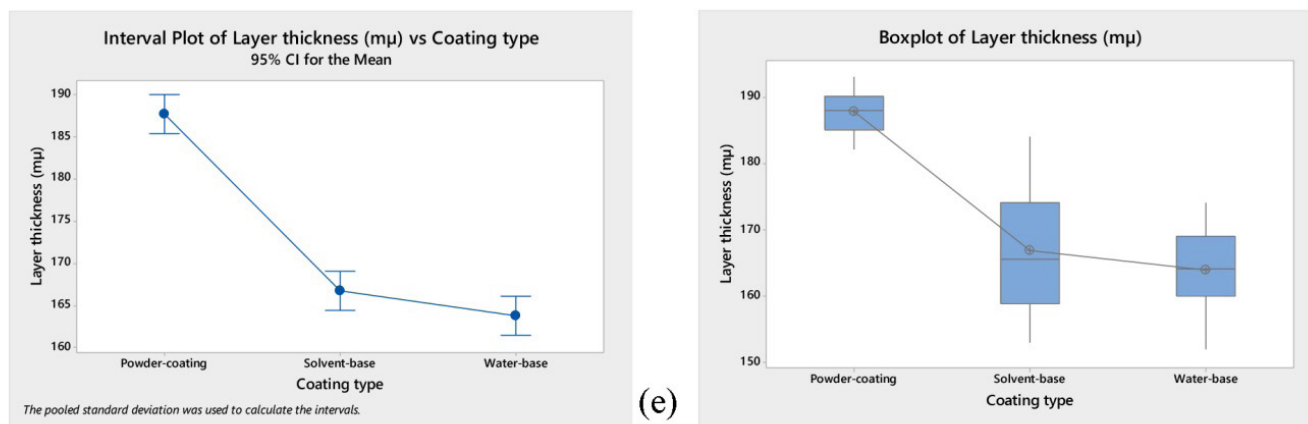

(f)

Figure 4: (a-b) Boxplot and interval plot results for adhesion strength (c-d) Boxplot and interval plot results for coating hardness (e-f) Boxplot and interval plot results for layer thickness. 


\section{Evaluation of the adhesion strength, layer thickness and coating hardness model}

Normality assumption is verified by applying the normal probability plot and histogram of residuals. From the (Figure 5a, Figure 5c and Figure 5e), as the residuals generally fall on a straight line implying, the errors were resembled the normal distribution. (Figure 5b, Figure 5d and Figure 5f) show standardized residuals, instead of ordinary, the residuals were applied to identify the outliers. Standardized residuals $d_{i}$ was computed by Equation 5:

$$
d_{i}=\frac{e_{i j}}{\sqrt{M S_{E}}}
$$

The term of $\sqrt{M S_{E}}$ is mean error sum of square. In (Figure 5b, Figure $5 \mathrm{~d}$ and Figure $5 \mathrm{f}$ ), standardized residuals values were found in interval $-3 \leq \mathrm{d}_{\mathrm{i}} \leq 3$. Therefore, the proposed approach was adequate and there was not any violation of the independence or constant variance assumption.
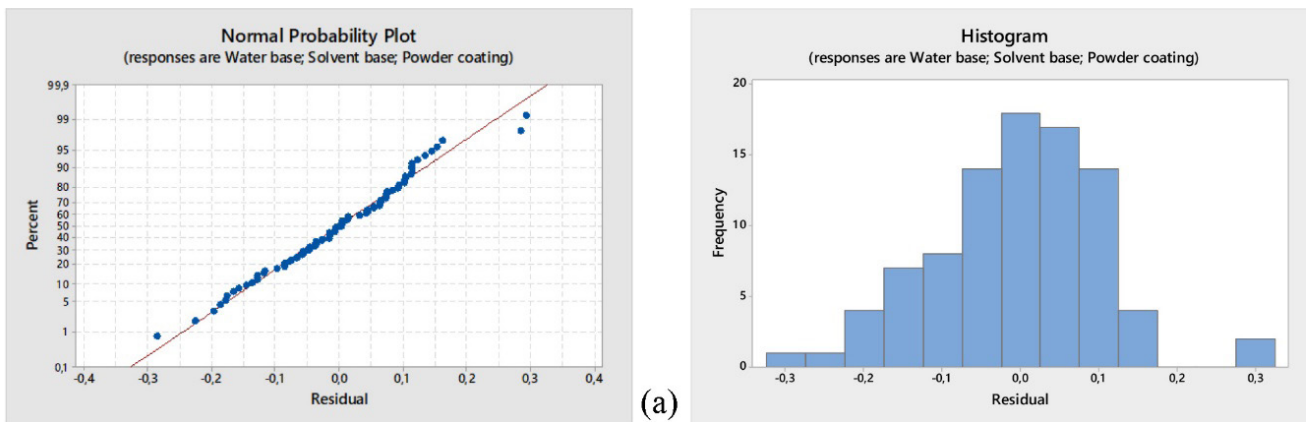

(b)
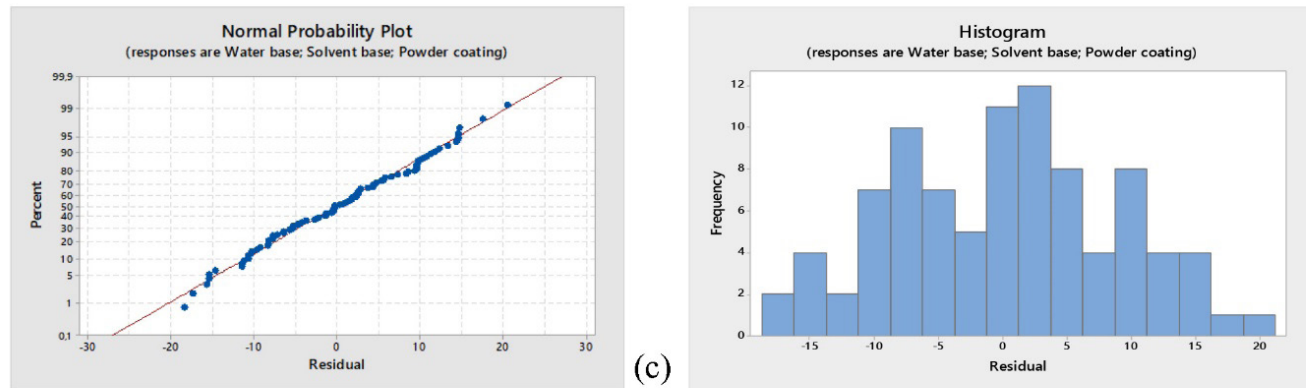

(d)
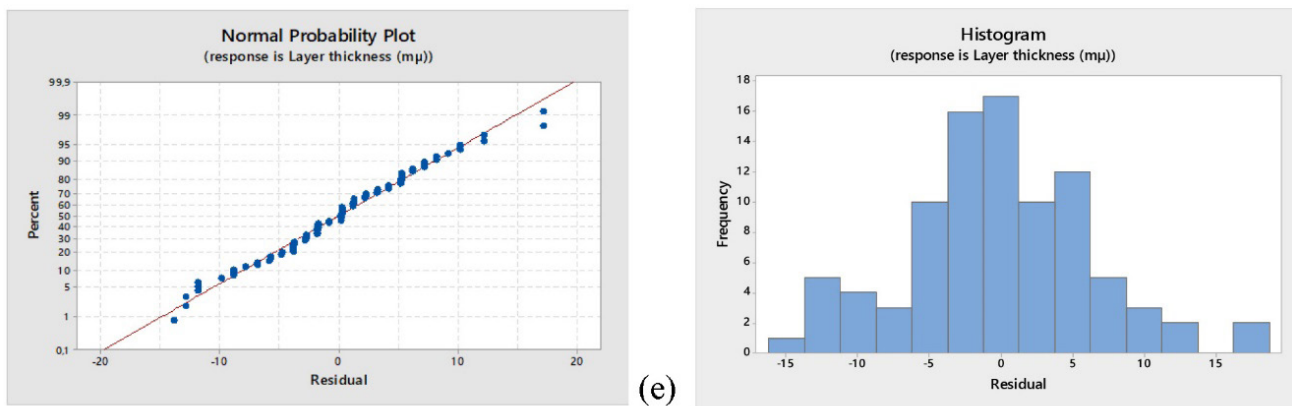

(e)

Figure 5: (a-c-e) Normal probability plot for standardized residuals (b-d-f) histogram of standardized residuals. 


\section{Analysis of rapid-deformation test}

Rapid-Deformation test was performed according to EN ISO 4211-4-2017. Steel ball with a diameter of $14 \mathrm{~mm}$ was dropped freely with a weight of $500 \mathrm{~g}$. from the standardized heights on the sample surface. The deformations were observed and the resistances were determined representing impact value from 1 to 5; 1 referring to holes, cracks and peel offs for non-resistant coating and 5 referring to no changes for resistant coating. The results of the evaluation using 30 samples are given in (Table 11).

Table 11: Results of rapid- deformation test.

\begin{tabular}{|c|c|c|c|}
\hline Sample of number & Solvent-based & Water-based & Powder-coating \\
\hline 1 & 4 & 4 & 4 \\
\hline 2 & 3 & 4 & 3 \\
\hline 3 & 5 & 4 & 4 \\
\hline 4 & 3 & 4 & 4 \\
\hline 5 & 4 & 3 & 4 \\
\hline 6 & 4 & 5 & 3 \\
\hline 7 & 5 & 4 & 4 \\
\hline 8 & 4 & 5 & 3 \\
\hline 9 & 4 & 4 & 3 \\
\hline 10 & 4 & 4 & \\
\hline
\end{tabular}

Kruskal-Wallis test result is given in Table 12. It was presented with results of sample size $(\mathrm{N})$, median, average of rank and Z-critical values. The results of average of rank for water-based, solvent-based and powder coating were 18,4;17,1 and 11,0 respectively. According to these results, it is found that the deformation in the powder coated surface is higher than the other coating types.

Table 12: Result of Kruskal-Wallis test.

\begin{tabular}{|c|c|c|c|c|}
\hline Paint type & $\mathbf{N}$ & Median & Ave Rank & $\mathbf{Z}$ \\
\hline Water-base & 10 & 4,000 & 18,4 & 1,28 \\
\hline Solvent-base & 10 & 4,000 & 17,1 & $-1,98$ \\
\hline Powder-coating & 10 & 3,500 & 11,0 & 0,70 \\
\hline Overall & 30 & & 15,5 & \\
\hline
\end{tabular}

\section{CONCLUSIONS}

In this study, MDF samples were coated with water-base, polyurethane-base and powder coating applications. Adhesion strength, surface coating hardness and impact deformations test were used to evaluate the surface coating performance. 
The results are as follows:

Grubb's test was used to find the outlier data. Analyze of the normally distribution data was performed by using normal probability plot. One-way ANOVA analysis was applied to determine the significant parameters. Tukey's test was used to determine the significance differences for each group. Rapid deformation test was evaluated with Kruskal-Wallis method.

The results of adhesion strength and surface coating hardness were analyzed by using ANOVA method. According to the results, coating type was the effective factor on strength adhesion, layer thickness and surface coating hardness. $R^{2}$ values of adhesion strength, layer hardness and film layer thickness were 93,60\%; 95,33 $\%$ and $73,90 \%$, respectively. Adj- $R^{2}$ values of adhesion strength, layer hardness and film layer thickness were $93,45 \% ; 95,23 \%$ and $73,30 \%$, respectively.

The highest layer thickness value was determined as powder coating application, while the lowest layer thickness was determined as water and solvent-based application. The reason for this situation, the solid content ratio of powder coatings was higher than solvent and water based coatings. Moreover, powder coated MDF surface was applied with primer and top coat.

The highest adhesion resistance was found as water-base coating application, while the lowest resistance was determined as solvent-based application. The highest surface coating hardness was resulted as powder coating application, while the lowest coating hardness was resulted as solvent-based application.

Coating type was the effective factor on the rapid-deformation test. The highest impact deformation resistance was found as water-based application, while the lowest resistance is found as powder coating application. The reason for this situation, water-based coating has more tough flexible than solvent based and powder coating.

From the powder coating application, surface coating hardness test results were higher than solvent and water based coating application. The reasons for this can be explained by the fact that the coating types have different chemical structures.

\section{ACKNOWLEDGMENTS}

The authors thank to TUBITAK (The Scientific and Technological Research Council of TURKEY) for support and contributions during the doctoral thesis.

\section{REFERENCES}

Acda, M.N.; Devera, E.E.; Cabangon, R.J.; Ramos, H.J. 2012. Effects of plasma modification on adhesion properties of wood. International Journal of Adhesion \& Adhesives 32(1):70-75. 176

Ahola, P. 1991. Adhesion between paint and wood substrate. Surface Coatings International 74(5):173-

Cakicier, N.; Korkut, S.; Korkut, D. 2011. Varnish layer hardness, scratch resistance, and glossiness of various wood species as affected by heat treatment. BioResources 6(2):1648-1658. 
Cool, J.; Hernández, R.E. 2011. Improving the sanding process of black spruce wood for surface quality and water-based coating adhesion. Forest Products Journal 61(5): 372-380.

Darmawan, W.; Nandika, D.; Noviyanti, E.; Alipraja, I.; Lumongga, D.; Gardnes, D.; Gerardin, P. 2018. Wettability and bonding quality of exterior coatings on jabon and sengon wood surfaces. J Coat Technol Res 15(1):95-104.

De Moura, L.F.; Hernandez, R.E. 2006. Effects of abrasive mineral, grit size and feed speed on the quality of sanded surfaces of sugar maple wood. Wood Sci Technol 40(6): 517-530.

Dilik, T.; Erdinler, S.; Hazir, E.; Koc, H.; Hiziroglu, S. 2015. Adhesion strength of wood based composites coated with cellulosic and polyurethane paints. Advances in Materials Science and Engineering 2015 (Article ID 745675) 1-5.

Erdinler, S.; Koç, H.;Dilik, T.; Hazir, E.2019. Layer thickness performances of coatings on MDF:Polyurethane and cellulosic paints. Maderas-Cienc Tecnol 21(3):317-326.

EN ISO 1522. 2016. Paints and Varnishes-Pendulum Damping Test. Turkish Standards Institution, Ankara, Turkey.

EN ISO 4624. 2016. Paints and Varnishes-Pull of test for adhesion. Turkish Standards Institution, Ankara, Turkey.

EN ISO 4211-4. 2017. Furniture-Tests for surfaces-Part 4.Assesment of resistance to impact.

Gurleyen, L.; Ayata, U.; Cakicier, N. 2017. Effect of heat treatment on the adhesion strength, pendulum hardness, surface roughness, color and glossiness of Scot pine laminated parquet with two different types of varnish application. Maderas-Cienc Tecnol 19(2):213-224.

Hernandez, R.E.; Cool, J. 2008. Evaluation of three surfacing methods on paper birch wood relation to water and solvent borne coatings performance. Wood Fiber Sci 40(3):459-469.

Jocham, C.; Schmidt, T.W.; Wuzella, G.; Teischinger, A.; Kandelbauer, A. 2012. Adhesion improvement of powder coating on medium density fiberboard (MDF) by thermal pre-treatment. Journal of Adhesion Science and Technology 25(15):1937-1946.

Keskin, H.; Tekin, A. 2011. Abrasion resistance of cellulosic, synthetic, polyurethane, waterborne and acidhardening varnishes used woods. Construction and Building Materials 25(2):638-643.

Landry, V.; Blanchet, P.; Cormier L.M. 2013. Water-based and solvent-based stains: Impact on the grain raising in Yellow Birch. BioResources 8(2):1997-2009.

Nejad, M.; Cooper, P. 2011. Exterior wood coatings. Part-2: modeling correlation between coating properties and their weathering performance. Journal of Coatings Technology Research 8(4):459-467.

Nejad, M.; Ung, T.; Cooper, P. 2012. Effect of coatings on ACQ preservative component distribution and solubility after natural weathering exposure. Wood Sci Technol 46(6):1169-1180.

Ramananantoandro, T.; Eyma, F.; Belloncle, C.; Rince, S.; Irle, M. 2018. Effect of machining parameters on raised grain occurring after the application of water-based finishes. European Journal of Wood and Wood Products 76(4):1323-1333.

Rield, B.; Angle, C.; Pregent, J.; Blanchet, P.; Stafford, L. 2014. Effect of wood surface modification by atmospheric-pressure plasma on waterborne coating adhesion. BioResources 9(3):4908-4923.

Salca, E.A.; Krystofiak, T.; Lis, B. 2017. Evaluation of selected properties of Alder wood as functions of sanding and coating. Coatings 7(10):176-191.

Salca, E.A.; Krystofiak, T.; Lis, B.; Mazela, B.; Proszyk, S. 2016. Some coating properties of black alder wood as function of varnish type and applications method. BioResources 11(3):7580-7594. 
Sogutlu, C.; Nzokou, P.; Koc, Ismail.; Tutgun, R.; Döngeş, N. 2016. The effect of surface roughness on varnish adhesion strength of wood materials. Journal of Coating Technology and Research 13(5):863-870. 